\title{
On the geometric quantization of twisted Poisson manifolds
}

\author{
Fani Petalidou \\ Faculty of Sciences and Technology \\ University of Peloponnese \\ 22100 Tripoli - Greece \\ E-mail: petalido@uop.gr
}

\begin{abstract}
We study the geometric quantization process for twisted Poisson manifolds. First, we introduce the notion of Lichnerowicz-twisted Poisson cohomology for twisted Poisson manifolds and we use it in order to characterize their prequantization bundles and to establish their prequantization condition. Next, we introduce a polarization and we discuss the quantization problem. In each step, several examples are presented.
\end{abstract}

Keywords: Twisted Poisson manifold, geometric quantization.

A.M.S. classification (2000): 53D50, 53D17.

\section{Introduction}

Geometric quantization is a useful procedure, founded in differential geometry, that allows us to understand the relation between classical and quantum mechanics by associating a quantum system to each classical system. This process consists of attaching to each classical system a complex Hilbert space and to each classical observable on the phase space of the classical system a quantum observable, i.e., a Hermitian operator on the Hilbert space, in such a way that the Poisson bracket of two classical observables is attached, up to a purely imaginary constant, with the commutator of the operators. It is completed in two steps: (i) the prequantization and (ii) the quantization. If $M$ is the phase space of the classical system equipped with a symplectic structure $\omega$, at the first step, one associates to $M$ a Hermitian line bundle $\pi: K \rightarrow M$ with a Hermitian connection having as curvature form the symplectic form $\omega . K$ is called the prequantization bundle of $(M, \omega)$ and exists under the prequantization condition: The cohomology class of $\omega$ is integral. Then, the Poisson Lie algebra $\left(C^{\infty}(M, \mathbb{R}),\{\cdot, \cdot\}\right)$ acts faithfully on the space of cross sections $\Gamma(K)$ of $\pi: K \rightarrow M$. At the second step, imposing a polarization, one constructs the Hilbert space $\mathcal{H}$ used in quantum mechanics out of $\Gamma(K)$ and one restricts the problem to a suitable Lie subalgebra of $\left(C^{\infty}(M, \mathbb{R}),\{\cdot, \cdot\}\right)$ that is represented irreducibly on $\mathcal{H}$. For a short introductive presentation of the subject, we can consult [14]. For a more extensive, but without too much detail, treatment of the problem, we suggest [2] where we can find a complete guide to the literature. We also refer, as standard references, the books [20] and [29].

The theory of geometric quantization was first developed for symplectic manifolds by B. Kostant [11] and J.M. Souriau [21], independently. Their approaches are different, but equivalent, and they have important applications. Later, it was extended 
by J. Huebschmann [6] to Poisson algebras and by I. Vaisman [24] (see, also [25]) to Poisson manifolds. In [6], the geometric quantization of Poisson manifolds appears as a particular case of the geometric quantization of Poisson algebras, while, in [24], this quantization is presented straightforwardly, using usual differential geometric techniques. In [13], Kostant's theory was adapted by M. de León et al. for Jacobi manifolds and, recently, by A. Weinstein and M. Zambon [28] for Dirac manifolds.

The purpose of the present paper is to study the geometric quantization problem for twisted Poisson manifolds. A such manifold $M$ is equipped with a bivector field $\Lambda$ of which the Schouten bracket with itself is equal to the image by $\Lambda^{\sharp}$ of a closed 3form $\varphi$ on $M$. These manifolds were introduced by P. Ševera and A. Weinstein in [18], under the name Poisson manifolds with 3-form background, stimulated by the works of J.S. Park [16], L. Cornalba and R. Schiappa [5], C. Klimčík and T. Ströbl [7] on deformation quantization and string theory in which such 3-forms played an important role. In order to understand the role of $\varphi$ on a twisted Poisson manifold $(M, \Lambda)$, one introduces on the space $C^{\infty}(M, \mathbb{R})$ of the real smooth functions on $M$ the bracket $\{f, g\}=\Lambda(d f, d g)$ and one looks its Jacobi identity which is true up to an extra term involving $\varphi$. Thus, $\left(C^{\infty}(M, \mathbb{R}),\{\cdot, \cdot\}\right)$ is no longer a Lie algebra. This result has an essential influence on the prequantization procedure of a twisted Poisson manifold as is explained in paragraph 4 .

The paper is organized as follows. In section 2 we recall the definition of a twisted Poisson manifold, we give some main examples of such manifolds and we introduce the Lichnerowicz-twisted Poisson cohomology. In section 3, the notion of twisted PoissonChern class of a complex line bundle over a twisted Poisson manifold is defined by using the concept of contravariant derivative given by I. Vaisman in [24]. Section 4 is devoted to the formulation of the integrality prequantization condition of a twisted Poisson manifold. Several interesting examples are discussed. Finally, in section 5, we develop the quantization process of a twisted Poisson manifold by introducing a polarization and we present a computational example.

We mention that the deformation quantization of twisted Poisson structures is discussed in the papers [19] and [1] with physical motivation. Also, we note that A. Weinstein and $\mathrm{P}$. Xu developed in [27] an alternative approach to the quantization problem of Poisson manifolds by using symplectic groupoids. We believe that we can extend their method to twisted Poisson manifolds by using twisted symplectic groupoids that are introduced in [4] by $\mathrm{A}$. Cattaneo and $\mathrm{P}$. $\mathrm{Xu}$. We postpone this study to a future paper.

\section{Twisted Poisson manifolds}

A twisted Poisson manifold is a differentiable manifold $M$ equipped with a bivector field $\Lambda$ and a closed 3 -form $\varphi$ on $M$, called the background 3 -form, such that

$$
\frac{1}{2}[\Lambda, \Lambda]=\Lambda^{\sharp}(\varphi) .
$$

In the above formula, $[\cdot, \cdot]$ denotes the Schouten bracket and $\Lambda^{\sharp}$ is the natural extension of $\Lambda^{\sharp}: \Gamma\left(T^{*} M\right) \rightarrow \Gamma(T M)$, given, for all $\alpha, \beta \in \Gamma\left(T^{*} M\right)$, by

$$
\left\langle\beta, \Lambda^{\sharp}(\alpha)\right\rangle=\Lambda(\alpha, \beta),
$$

to a homomorphism from $\Gamma\left(\bigwedge^{k} T^{*} M\right)$ to $\Gamma\left(\bigwedge^{k} T M\right), k \in \mathbb{N}$, defined, for all $\eta \in$ $\Gamma\left(\bigwedge^{k} T^{*} M\right)$ and $\alpha_{1}, \ldots, \alpha_{k} \in \Gamma\left(T^{*} M\right)$, by

$$
\Lambda^{\sharp}(\eta)\left(\alpha_{1}, \ldots, \alpha_{k}\right)=(-1)^{k} \eta\left(\Lambda^{\sharp}\left(\alpha_{1}\right), \ldots, \Lambda^{\sharp}\left(\alpha_{k}\right)\right)
$$


and, for any $f \in C^{\infty}(M, \mathbb{R})$, by $\Lambda^{\sharp}(f)=f$. In the following, a twisted Poisson manifold will be denoted by the triple $(M, \Lambda, \varphi)$.

\subsection{Examples of twisted Poisson manifolds}

1) Poisson manifolds: Let $(M, \Lambda)$ be a Poisson manifold, i.e., $[\Lambda, \Lambda]=0$, and $\varphi$ a closed 3 -form on $M$ satisfying $\Lambda^{\sharp}(\varphi)=0$. Then, $(M, \Lambda, \varphi)$ is a twisted Poisson manifold. This happens for 3-dimensional Poisson manifolds. Since $\operatorname{Im} \Lambda^{\sharp}$ defines a foliation of $M$ whose the leaves are of dimension 0 or 2 , we have that any three sections of $\operatorname{Im} \Lambda^{\sharp}$ are linearly dependent on $M$. Thus, any 3 -form $\varphi$ on $M$ is closed and $\Lambda^{\sharp}(\varphi)=0$.

2) Twisted Poisson manifolds associated to symplectic manifolds I: Let $\left(M_{0}, \omega_{0}\right)$ be a symplectic manifold of dimension $2 n, n \geq 2$, and $\Lambda_{0}$ the unique bivector field on $M_{0}$ given, for all $\alpha \in \Gamma\left(T^{*} M_{0}\right)$, by $i\left(\Lambda_{0}^{\sharp}(\alpha)\right) \omega_{0}=-\alpha$, i.e., $\Lambda_{0}=\Lambda_{0}^{\sharp}\left(\omega_{0}\right)$. Then, for any non constant function $f \in C^{\infty}\left(M_{0}, \mathbb{R}\right)$, the bivector field $\Lambda=f \Lambda_{0}$ and the closed 3form $\varphi=-f^{-2} \omega_{0} \wedge d f$ define a twisted Poisson structure on $M_{0}$. In fact, by a simple computation, we find

$$
\begin{aligned}
\frac{1}{2}[\Lambda, \Lambda] & =\frac{1}{2}\left[f \Lambda_{0}, f \Lambda_{0}\right]=-\left(f \Lambda_{0}\right) \wedge \Lambda_{0}^{\sharp}(d f) \\
& =\left(f \Lambda_{0}\right)^{\sharp}\left(-f^{-2} \omega_{0} \wedge d f\right)=\Lambda^{\sharp}(\varphi) .
\end{aligned}
$$

3) Twisted Poisson manifolds associated to symplectic manifolds II: Let $\left(M_{0}, \omega_{0}\right)$ be a $2 n$-dimensional symplectic manifold with $n \geq 2$ and $\Lambda_{0}$ the nondegenerate Poisson structure defined by $\omega_{0}$ as in Example 2. Then, the triple $(M, \Lambda, \varphi)$, where $M=M_{0} \times \mathbb{R}$,

$$
\Lambda=e^{t}\left(\Lambda_{0}+\Lambda_{0}^{\sharp}(d f) \wedge \frac{\partial}{\partial t}\right) \quad \text { and } \quad \varphi=-e^{-t} \omega_{0} \wedge d t,
$$

$t$ being the canonical coordinate on $\mathbb{R}$ and $f \in C^{\infty}\left(M_{0}, \mathbb{R}\right)$, is a twisted Poisson manifold. We have

$$
\begin{aligned}
\frac{1}{2}[\Lambda, \Lambda] & =\frac{1}{2}\left[e^{t}\left(\Lambda_{0}+\Lambda_{0}^{\sharp}(d f) \wedge \frac{\partial}{\partial t}\right), e^{t}\left(\Lambda_{0}+\Lambda_{0}^{\sharp}(d f) \wedge \frac{\partial}{\partial t}\right)\right] \\
& =e^{2 t} \Lambda_{0}^{\sharp}(d f) \wedge \Lambda_{0}=\Lambda^{\sharp}\left(-e^{-t} \omega_{0} \wedge d t\right)=\Lambda^{\sharp}(\varphi) .
\end{aligned}
$$

4) Twisted Poisson manifolds associated to Poisson manifolds: Let $\left(M, \Lambda_{0}, \omega\right)$ be a Poisson manifold endowed with a 2-form $\omega$ such that the operator $I d+\omega^{b} \circ \Lambda_{0}^{\sharp}$ : $T^{*} M \rightarrow T^{*} M$ is invertible. Then, the vector bundle map $\Lambda^{\sharp}=\Lambda_{0}^{\sharp} \circ\left(I d+\omega^{b} \circ \Lambda_{0}^{\sharp}\right)^{-1}$ defines a $(-d \omega)$-twisted Poisson structure on $M$. (For more details, see [18].)

5) Twisted Poisson structures induced by twisted Jacobi manifolds: Let $(M, \Lambda, E, \omega)$ be a twisted Jacobi manifold ([15]), i.e., $M$ is a differentiable manifold endowed with a bivector field $\Lambda$, a vector field $E$ and a 2 -form $\omega$ such that

$$
\frac{1}{2}[\Lambda, \Lambda]+E \wedge \Lambda=\Lambda^{\sharp}(d \omega)+\left(\Lambda^{\sharp} \omega\right) \wedge E
$$

and

$$
[E, \Lambda]=\left(\Lambda^{\sharp} \otimes 1\right)(d \omega)(E)-\left(\left(\Lambda^{\sharp} \otimes 1\right)(\omega)(E)\right) \wedge E .
$$

In $(5),\left(\Lambda^{\sharp} \otimes 1\right)(d \omega)$ and $\left(\Lambda^{\sharp} \otimes 1\right)(\omega)$ denote, respectively, the sections of $\left(\bigwedge^{2} T M\right) \otimes T^{*} M$ and $T M \otimes T^{*} M$ that act on multivector fields by contraction with the factor in $T^{*} M$ (see, [15]). We consider a submanifold $M_{0}$ of $M$, of codimension 1 and transverse to 
$E$. Let $\varpi: U \rightarrow M_{0}$ be the projection on $M_{0}$ of a tubular neighbourhood $U$ of $M_{0}$ in $M$ such that, for any $x \in M_{0}, \varpi^{-1}(x)$ is a connected arc of the integral curve of $E$ through $x$. If $\omega=\varpi^{*} \omega_{0}$, where $\omega_{0}$ is a 2 -form on $M_{0}$, then, the twisted Jacobi structure $(\Lambda, E, \omega)$ of $M$ induces a twisted Poisson structure $\left(\Lambda_{0}, \varphi_{0}\right)$ on $M_{0}$, where $\Lambda_{0}=\varpi_{*} \Lambda$ and $\varphi_{0}=d \omega_{0}$. In fact, by projecting (4) along the integral curves of $E$, we get

$$
\frac{1}{2}\left[\Lambda_{0}, \Lambda_{0}\right]=\Lambda_{0}^{\sharp}\left(d \omega_{0}\right),
$$

while the projection of (5) is annihilated identically. (For more details, see [17]).

\subsection{The Lichnerowicz-twisted Poisson cohomology of a twisted Pois- son manifold}

Let $(M, \Lambda, \varphi)$ be a twisted Poisson manifold. As in the case of a Poisson manifold, we introduce hamiltonian vector fields on $M$ by setting, for any $f \in C^{\infty}(M, \mathbb{R}), X_{f}=$ $\Lambda^{\sharp}(d f)$ and we define on $C^{\infty}(M, \mathbb{R})$ the internal composition law

$$
\{f, g\}=\Lambda(d f, d g), \quad f, g \in C^{\infty}(M, \mathbb{R}),
$$

that is bilinear and skew-symmetric but its Jacobi identity is modified by $\varphi$ :

$$
\{f,\{g, h\}\}+\{g,\{h, f\}\}+\{h,\{f, g\}\}=\Lambda^{\sharp}(\varphi)(d f, d g, d h) .
$$

Therefore, $\left(C^{\infty}(M, \mathbb{R}),\{\cdot, \cdot\}\right)$ is no longer a Lie algebra. In this paper, we will say that it is a $\varphi$-twisted Lie algebra. Since the Jacobi identity is violated, we cannot, in general, define the Chevalley-Eilenberg cohomology of $\left(C^{\infty}(M, \mathbb{R}),\{\cdot, \cdot\}\right)$ relative to the representation defined by the hamiltonian vector fields, i.e., to the representation given by

$$
C^{\infty}(M, \mathbb{R}) \times C^{\infty}(M, \mathbb{R}) \rightarrow C^{\infty}(M, \mathbb{R}), \quad(f, g) \rightarrow X_{f}(g) .
$$

However, any twisted Poisson structure $(\Lambda, \varphi)$ on $M$ produces a Lie algebroid structure on the cotangent bundle $T^{*} M$ of $M$, as in the ordinary case. The Lie bracket on the space of smooth sections of $T^{*} M$ is given, for any $\alpha, \beta \in \Gamma\left(T^{*} M\right)$, by

$$
\{\alpha, \beta\}^{\varphi}=\{\alpha, \beta\}+\varphi\left(\Lambda^{\sharp}(\alpha), \Lambda^{\sharp}(\beta), \cdot\right),
$$

where $\{\cdot, \cdot\}$ denotes the Koszul bracket $([12])$ associated to $\Lambda$, i.e.

$$
\{\alpha, \beta\}=\mathcal{L}_{\Lambda^{\sharp}(\alpha)} \beta-\mathcal{L}_{\Lambda^{\sharp}(\beta)} \alpha-d \Lambda(\alpha, \beta),
$$

and characterized by $\{d f, d g\}=d\{f, g\}$ and the Leibniz identity $\{\alpha, f \beta\}=f\{\alpha, \beta\}+$ $\left(\mathcal{L}_{\Lambda^{\sharp}(\alpha)} f\right) \beta$. The anchor map is the vector bundle map $\Lambda^{\sharp}: T^{*} M \rightarrow T M$ defined by (2), while, the exterior derivative operator $\partial_{\varphi}$ on $\Gamma(\bigwedge T M)$ determined by $\left(\{\cdot, \cdot\}^{\varphi}, \Lambda^{\sharp}\right)$ is defined, for all $P \in \Gamma\left(\bigwedge^{k} T M\right)$ and $\alpha_{1}, \ldots, \alpha_{k+1} \in \Gamma\left(T^{*} M\right)$, by

$$
\begin{aligned}
\partial_{\varphi} P\left(\alpha_{1}, \ldots, \alpha_{k+1}\right)= & \sum_{i=1}^{k+1}(-1)^{i+1} \Lambda^{\sharp}\left(\alpha_{i}\right)\left(P\left(\alpha_{1}, \ldots, \hat{\alpha}_{i}, \ldots, \alpha_{k+1}\right)\right) \\
& +\Sigma_{1 \leq i<j \leq k+1}(-1)^{i+j} P\left(\left\{\alpha_{i}, \alpha_{j}\right\}^{\varphi}, \alpha_{1}, \ldots, \hat{\alpha}_{i}, \ldots, \hat{\alpha}_{j}, \ldots, \alpha_{k+1}\right),
\end{aligned}
$$

where the hat denotes missing arguments. Since $\partial_{\varphi}^{2}=0,\left(\Gamma(\bigwedge T M), \partial_{\varphi}\right)$ is a chain complex. 
Definition 2.1 We call Lichnerowicz-twisted Poisson cohomology (L-tP cohomology) of $(M, \Lambda, \varphi)$ the cohomology of $\left(\Gamma(\bigwedge T M), \partial_{\varphi}\right)$. It is denoted by $H_{L-t P}^{*}(M, \Lambda, \varphi)$ or, for simplicity, $H_{L-t P}^{*}(M)$ and, for any $k \in \mathbb{N}$,

$$
H_{L-t P}^{k}(M)=\frac{\operatorname{ker}\left(\partial_{\varphi}: \bigwedge^{k} T M \rightarrow \bigwedge^{k+1} T M\right)}{\operatorname{Im}\left(\partial_{\varphi}: \bigwedge^{k-1} T M \rightarrow \bigwedge^{k} T M\right)},
$$

with the convention $\bigwedge^{-1} T M=\{0\}$. The cohomology class of any element $P \in \operatorname{ker}\left(\partial_{\varphi}\right.$ : $\left.\bigwedge^{k} T M \rightarrow \bigwedge^{k+1} T M\right)$ is denoted $[P]^{\varphi}$.

By a simple, but long, computation, we can prove that the homomorphism $\Lambda^{\sharp}$ : $\Gamma\left(\bigwedge^{*} T^{*} M\right) \rightarrow \Gamma\left(\bigwedge^{*} T M\right)$ is a chain map, namely,

$$
\partial_{\varphi} \circ \Lambda^{\sharp}=-\Lambda^{\sharp} \circ d .
$$

Hence, we deduce

Proposition 2.2 If $H_{d R}^{*}(M, \mathbb{R})$ is the de Rham cohomology of $(M, \Lambda, \varphi)$, the homomorphism of complexes $\Lambda^{\sharp}:\left(\Gamma\left(\Lambda^{*} T^{*} M\right), d\right) \rightarrow\left(\Gamma\left(\Lambda^{*} T M\right), \partial_{\varphi}\right)$ induces a homomorphism in cohomology, also denoted by $\Lambda^{\sharp}$,

$$
\begin{aligned}
& \Lambda^{\sharp}: H_{d R}^{*}(M, \mathbb{R}) \rightarrow H_{L-t P}^{*}(M) \\
& {[\alpha] \quad \mapsto \quad\left[\Lambda^{\sharp}(\alpha)\right]^{\varphi} .}
\end{aligned}
$$

If $\Lambda$ is nondegenerate, then (9) is an isomorphism.

\section{Twisted Poisson-Chern class of a complex line bundle over a twisted Poisson manifold}

Let $(M, \Lambda, \varphi)$ be a twisted Poisson manifold, $\pi: K \rightarrow M$ a complex line bundle over $M, \Gamma(K)$ the space of the global cross sections of $\pi: K \rightarrow M$ and $\operatorname{End}_{\mathbb{C}}(\Gamma(K))$ the space of the complex linear endomorphisms of $\Gamma(K)$.

Definition 3.1 $A$ contravariant derivative $D$ on $\pi: K \rightarrow M$ is a $\mathbb{R}$-linear mapping

$$
D: \Gamma\left(T^{*} M\right) \rightarrow \operatorname{End}_{\mathbb{C}}(\Gamma(K))
$$

i.e., for any $\alpha, \beta \in \Gamma\left(T^{*} M\right)$ and $f \in C^{\infty}(M, \mathbb{R})$,

$$
D_{\alpha+\beta}=D_{\alpha}+D_{\beta} \quad \text { and } \quad D_{f \alpha}=f D_{\alpha}
$$

such that

$$
D_{\alpha}(f s)=f D_{\alpha} s+\left(\Lambda^{\sharp}(\alpha) f\right) s, \quad \text { for all } s \in \Gamma(K) .
$$

We say that $D$ is Hermitian or compatible with a Hermitian metric $h$ on $\pi: K \rightarrow M$, if, for all $\alpha \in \Gamma\left(T^{*} M\right)$ and $s_{1}, s_{2} \in \Gamma(K)$,

$$
\Lambda^{\sharp}(\alpha)\left(h\left(s_{1}, s_{2}\right)\right)=h\left(D_{\alpha} s_{1}, s_{2}\right)+h\left(s_{1}, D_{\alpha} s_{2}\right) .
$$

We note that such (Hermitian) operators on $\pi: K \rightarrow M$ always exist; it suffices to consider an arbitrary (Hermitian) connection $\nabla$ on $\pi: K \rightarrow M$ and to put $D_{\alpha}=\nabla_{\Lambda^{\sharp}(\alpha)}$. 
Definition 3.2 The curvature $C_{D}$ of a contravariant derivative $D$ on $\pi: K \rightarrow M$ is the mapping

$$
C_{D}: \Gamma\left(T^{*} M\right) \times \Gamma\left(T^{*} M\right) \rightarrow \operatorname{End}_{\mathbb{C}}(\Gamma(K))
$$

defined, for all $\alpha, \beta \in \Gamma\left(T^{*} M\right)$, by

$$
C_{D}(\alpha, \beta)=D_{\alpha} \circ D_{\beta}-D_{\beta} \circ D_{\alpha}-D_{\{\alpha, \beta\}} .
$$

Proposition 3.3 $C_{D}$ is bilinear over $C^{\infty}(M, \mathbb{R})$ and skew-symmetric, i.e.,

$$
C_{D}(\alpha, \beta)=-C_{D}(\beta, \alpha), \quad \text { for all } \alpha, \beta \in \Gamma\left(T^{*} M\right) .
$$

Proof. The skew-symmetry of $C_{D}$ is an immediate consequence of its definition (13). Its bilinearity can be proved by using the linearity (10) and the property (11) of $D$.

Thus, from the above results and the fact that $\pi: K \rightarrow M$ is a complex line bundle over $M$, we have that there exists a globally defined complex bivector field $\Pi=\Pi_{1}+i \Pi_{2}$ on $M$, with $\Pi_{1}, \Pi_{2} \in \Gamma\left(\bigwedge^{2} T M\right)$, such that, for all $\alpha, \beta \in \Gamma\left(T^{*} M\right)$ and $s \in \Gamma(K)$,

$$
C_{D}(\alpha, \beta)(s)=\Pi(\alpha, \beta) s .
$$

For more details, we can consult [11] and adapt its results in the contravariant framework.

We extend, by linearity, the cohomology operator $\partial_{\varphi}$ on the complex multivector fields on $M$ by setting, for any $P \in \Gamma\left(\bigwedge^{k} T_{\mathbb{C}} M\right), P=P_{1}+i P_{2}$ with $P_{1}, P_{2} \in \Gamma\left(\bigwedge^{k} T M\right)$,

$$
\partial_{\varphi} P=\partial_{\varphi} P_{1}+i \partial_{\varphi} P_{2}
$$

Clearly, $\partial_{\varphi}^{2}=0$. Consequently, $\left(\Gamma\left(\bigwedge T_{\mathbb{C}} M\right), \partial_{\varphi}\right)$ is a chain complex whose cohomology will be called the complex Lichnerowicz-twisted Poisson cohomology of $(M, \Lambda, \varphi)$ and will be denoted by $H_{\mathbb{C} L-t P}^{*}(M, \Lambda, \varphi)$ or $H_{\mathbb{C} L-t P}^{*}(M)$.

Theorem 3.4 Let $\pi: K \rightarrow M$ be a complex line bundle over a twisted Poisson manifold $(M, \Lambda, \varphi), D$ a contravariant derivative on $\pi: K \rightarrow M, C_{D}$ the curvature of $D$ and $\Pi$ the complex bivector field on $M$ associated to $C_{D}$ (14). Then:

(i) $\Pi$ defines a cohomology class $[\Pi]^{\varphi}$ in $H_{\mathbb{C} L-t P}^{2}(M)$.

(ii) $[\Pi]^{\varphi}$ does not depend of the contravariant derivative $D$.

(iii) In the case where $D$ is compatible with a Hermitian metric $h$ on $\pi: K \rightarrow M$, $\Pi$ is purely imaginary.

Proof. (i) Let $s$ be a nowhere vanishing local section of $\pi: K \rightarrow M$. Since the complex dimension of the fibre of $\pi: K \rightarrow M$ is 1, we may associate to $s$ a unique complex local vector field on $M$ as follows. It is clear that, for any 1-form $\alpha$ on $M, \frac{D_{\alpha} s}{s}$ is a complex function on $M$ and the application $\alpha \mapsto \frac{D_{\alpha} s}{s}$ is $\mathbb{C}$-linear (10). Hence, there exists a unique complex local vector field $X=X_{1}+i X_{2}$ on $M$, with $X_{1}, X_{2}$ local real vector fields on $M$, such that, for all $\alpha \in \Gamma\left(T^{*} M\right)$,

$$
D_{\alpha} s=\langle\alpha, X\rangle s \text {. }
$$

We have that

$$
\Pi=\partial_{\varphi} X
$$


Effectively, for all $\alpha, \beta \in \Gamma\left(T^{*} M\right)$,

$$
\begin{array}{rll}
\Pi(\alpha, \beta) s \stackrel{\stackrel{(14)}{=}}{ } & C_{D}(\alpha, \beta)(s) \\
\stackrel{(13)}{=} & \left(D_{\alpha} \circ D_{\beta}-D_{\beta} \circ D_{\alpha}-D_{\{\alpha, \beta\}^{\varphi}}\right)(s) \\
\stackrel{(15)}{=} & D_{\alpha}(\langle\beta, X\rangle s)-D_{\beta}(\langle\alpha, X\rangle s)-\left\langle\{\alpha, \beta\}^{\varphi}, X\right\rangle s \\
\stackrel{(11)(15)}{=} & \langle\beta, X\rangle\langle\alpha, X\rangle s+\Lambda^{\sharp}(\alpha)(\langle\beta, X\rangle) s \\
& -\langle\alpha, X\rangle\langle\beta, X\rangle s-\Lambda^{\sharp}(\beta)(\langle\alpha, X\rangle) s-\left\langle\{\alpha, \beta\}^{\varphi}, X\right\rangle s \\
= & \partial_{\varphi} X(\alpha, \beta) s .
\end{array}
$$

Consequently, $\partial_{\varphi} \Pi=\partial_{\varphi}^{2} X=0$ which means that $\Pi$ defines a cohomology class in $H_{\mathbb{C} L-t P}^{2}(M)$ denoted by $[\Pi]^{\varphi}$.

(ii) Let $\tilde{D}$ be another contravariant derivative on $\pi: K \rightarrow M$ having curvature $C_{\tilde{D}}$ and $\tilde{X}$ the corresponding local complex vector field (see, (i)). We denote by $\tilde{\Pi}$ the corresponding to $C_{\tilde{D}}$ global complex bivector field on $M(14)$. From (16), we obtain

$$
\tilde{\Pi}-\Pi=\partial_{\varphi} \tilde{X}-\partial_{\varphi} X \Leftrightarrow \tilde{\Pi}=\Pi+\partial_{\varphi}(\tilde{X}-X) .
$$

Now, for any $\alpha \in \Gamma\left(T^{*} M\right)$, we define the mapping

$$
\hat{D}_{\alpha}=\tilde{D}_{\alpha}-D_{\alpha}: \Gamma(K) \rightarrow \Gamma(K)
$$

that is $\mathbb{C}$-linear. Therefore, there exists a globally defined complex vector field $\hat{X}$ on $M$ such that, for all $s \in \Gamma(K)$,

$$
\hat{D}_{\alpha} s=\langle\alpha, \hat{X}\rangle s .
$$

From the last two relations, we deduce that, in the overlapping of $X$ and $\tilde{X}$,

$$
\hat{X}=\tilde{X}-X
$$

So, using (18) in (17), we obtain $\tilde{\Pi}=\Pi+\partial_{\varphi} \hat{X}$, which means that $[\tilde{\Pi}]^{\varphi}=[\Pi]^{\varphi}$.

(iii) We assume that $D$ is compatible with a Hermitian metric $h$ on $\pi: K \rightarrow M$ and let $(e)$ be a local orthonormal basis of $\Gamma(K)$. Then, for all $\alpha \in \Gamma\left(T^{*} M\right)$, (12) gives us

$$
\begin{gathered}
\Lambda^{\sharp}(\alpha)(h(e, e))=h\left(D_{\alpha} e, e\right)+h\left(e, D_{\alpha} e\right) \Leftrightarrow 0 \stackrel{(15)}{=} h(\langle\alpha, X\rangle e, e)+h(e,\langle\alpha, X\rangle e) \Leftrightarrow \\
0=\langle\alpha, X\rangle+\overline{\langle\alpha, X\rangle} \Leftrightarrow 0=X+\bar{X},
\end{gathered}
$$

where the bar denotes complex conjugation. Hence, $X$ is purely imaginary and, because $\Pi=\partial_{\varphi} X$, we conclude that $\Pi$ is purely imaginary.

From the above theorem we get the following definition.

Definition 3.5 Let $\pi: K \rightarrow M$ be a complex line bundle over a twisted Poisson manifold $(M, \Lambda, \varphi), D$ a contravariant derivative on $\pi: K \rightarrow M$ having curvature $C_{D}$ whose the associated bivector field $\Pi$ is purely imaginary. Then, the well-defined cohomology class $\left[\frac{i}{2 \pi} \Pi\right]^{\varphi} \in H_{L-t P}^{2}(M)$ will be called the first real twisted Poisson-Chern class of $\pi: K \rightarrow M$.

Next, we will prove that $\left[\frac{i}{2 \pi} \Pi\right]^{\varphi}$ is the image by the homomorphism (9) of the usual first real Chern class of $\pi: K \rightarrow M$. 
We recall that, given a complex Hermitian line bundle $\pi: K \rightarrow M$ over a smooth manifold $M$, the first real Chern class of $\pi: K \rightarrow M$ is an element of the second de Rham cohomology of $M$ with integer coefficients and it is denoted $c_{1}(K, \mathbb{R}),[8]$. On the other hand, if $\pi: K \rightarrow M$ is endowed with a Hermitian connection $\nabla$ with curvature $C_{\nabla}$, i.e., for all $X, Y \in \Gamma(T M)$,

$$
C_{\nabla}(X, Y)=\nabla_{X} \circ \nabla_{Y}-\nabla_{Y} \circ \nabla_{X}-\nabla_{[X, Y]}
$$

there exists a purely imaginary closed 2-form $\Omega$ on $M([8])$ such that, for all $s \in \Gamma(K)$,

$$
C_{\nabla}(X, Y)(s)=\Omega(X, Y) s
$$

and, in this case, the first real Chern class $c_{1}(K, \mathbb{R})$ of $\pi: K \rightarrow M$ is just ([11]) the integral cohomology class $\left[\frac{i}{2 \pi} \Omega\right]$ in $H_{d R}^{2}(M, \mathbb{R})$. We note that $([11])$ the canonical injection $\varepsilon: \mathbb{Z} \rightarrow \mathbb{R}$ induces a homomorphism

$$
\varepsilon: H_{d R}^{2}(M, \mathbb{Z}) \rightarrow H_{d R}^{2}(M, \mathbb{R})
$$

and a class $[\alpha] \in H_{d R}^{2}(M, \mathbb{R})$ is called integral if it lies in the image $\operatorname{Im} \varepsilon$ of $\varepsilon$.

Theorem 3.6 Let $\pi: K \rightarrow M$ be a complex Hermitian line bundle over a twisted Poisson manifold $(M, \Lambda, \varphi), \nabla$ a Hermitian connection on $\pi: K \rightarrow M$ and $D$ the associated to $\nabla$ Hermitian contravariant derivative on $\pi: K \rightarrow M$, i.e., for any $\alpha \in$ $\Gamma\left(T^{*} M\right), D_{\alpha}=\nabla_{\Lambda^{\sharp}(\alpha)}$. If $c_{1}(K, \mathbb{R})$ and $\left[\frac{i}{2 \pi} \Pi\right]^{\varphi}$ are, respectively, the first real Chern class and the first real twisted Poisson-Chern class of $\pi: K \rightarrow M$, then

$$
\Lambda^{\sharp}\left(c_{1}(K, \mathbb{R})\right)=\left[\frac{i}{2 \pi} \Pi\right]^{\varphi},
$$

where $\Lambda^{\sharp}: H_{d R}^{2}(M, \mathbb{R}) \rightarrow H_{L-t P}^{2}(M)$ is the homomorphism (9) between the second de Rham cohomology and the corresponding Lichnerowicz-twisted Poisson cohomology of $M$.

Proof. Let $\omega$ be the local, purely imaginary, connection 1-form on $M$ associated to $\nabla$ ([11]) as follows. For any nowhere vanishing local section $s$ of $\pi: K \rightarrow M$ and any $Y \in \Gamma(T M)$,

$$
\nabla_{Y} s=\langle\omega, Y\rangle s .
$$

Then, the purely imaginary closed 2 -form $\Omega$ on $M$ associated to $C_{\nabla}$ (19) coincides with $d \omega$ (see, [8]) and $c_{1}(K, \mathbb{R})=\left[\frac{i}{2 \pi} \Omega\right]=\left[\frac{i}{2 \pi} d \omega\right]$. Moreover, if $X$ is the local purely imaginary vector field on $M$ defined by (15), from the definition of $D$, we get that, for any $\alpha \in \Gamma\left(T^{*} M\right)$,

$$
\begin{array}{rll}
D_{\alpha} s=\nabla_{\Lambda^{\sharp}(\alpha)} s & \stackrel{(15)(20)}{\Leftrightarrow} & \langle\alpha, X\rangle s=\left\langle\omega, \Lambda^{\sharp}(\alpha)\right\rangle s \\
& \Leftrightarrow & \langle\alpha, X\rangle s=-\left\langle\alpha, \Lambda^{\sharp}(\omega)\right\rangle s \\
& \Leftrightarrow & X=-\Lambda^{\sharp}(\omega) .
\end{array}
$$

Thus, if $\Pi$ is the purely imaginary bivector field on $M$ associated to the curvature $C_{D}$ of $D(14)$, we have

$$
\Pi \stackrel{(16)}{=} \partial_{\varphi} X \stackrel{(21)}{=}-\partial_{\varphi} \Lambda^{\sharp}(\omega) \stackrel{(8)}{=} \Lambda^{\sharp}(d \omega) .
$$

Consequently,

$$
\left[\frac{i}{2 \pi} \Pi\right]^{\varphi}=\left[\frac{i}{2 \pi} \Lambda^{\sharp}(d \omega)\right]^{\varphi} \stackrel{(9)}{=} \Lambda^{\sharp}\left(\left[\frac{i}{2 \pi} d \omega\right]\right)=\Lambda^{\sharp}\left(c_{1}(K, \mathbb{R})\right) .
$$




\section{Prequantization of twisted Poisson manifolds}

In this section, we will prequantize a twisted Poisson manifold $(M, \Lambda, \varphi)$ by associating to each differentiable function on $M$ an operator that acts on the space of cross sections of a Hermitian line bundle $\pi: K \rightarrow M$. As we have mentioned in Introduction, this approach was first developed by B. Kostant [11] and J.M. Souriau [21] for symplectic manifolds and was extended by J. Huebschmann [6] and I. Vaisman [24] to Poisson manifolds, by M. de León et al. [13] to Jacobi manifolds and by A. Weinstein and M. Zambon [28] to Dirac manifolds.

Let $(M, \Lambda, \varphi)$ be a twisted Poisson manifold and $\pi: K \rightarrow M$ a Hermitian line bundle over $M$ endowed with a contravariant derivative $D$ whose curvature is $C_{D}$. We define a representation ${ }^{-}$of the $\varphi$-twisted Lie algebra $\left(C^{\infty}(M, \mathbb{R}),\{\cdot, \cdot\}\right)$ on $\operatorname{End}_{\mathbb{C}}(\Gamma(K))$ by associating to each $f \in C^{\infty}(M, \mathbb{R})$ a complex endomorphism $\hat{f}$ of $\Gamma(K)$ that is defined, for any $s \in \Gamma(K)$, by

$$
\hat{f}(s)=D_{d f} s+2 \pi i f s .
$$

Since $\left(C^{\infty}(M, \mathbb{R}),\{\cdot, \cdot\}\right)$ is not a Lie algebra, the map

$$
\begin{array}{ccc}
\uparrow: C^{\infty}(M, \mathbb{R}) & \rightarrow & \operatorname{End}_{\mathbb{C}}(\Gamma(K)) \\
f & \mapsto & \hat{f}
\end{array}
$$

is no longer a homomorphism between $\left(C^{\infty}(M, \mathbb{R}),\{\cdot, \cdot\}\right)$ and $\left(\operatorname{End}_{\mathbb{C}}(\Gamma(K)),[\cdot, \cdot]\right)$, where $[\cdot, \cdot]$ denotes the usual commutator on $\operatorname{End}_{\mathbb{C}}(\Gamma(K))$, as the prequantization process requires. For this reason, we consider the subspace

$$
A=\left\{\hat{f} \in \operatorname{End}_{\mathbb{C}}(\Gamma(K)) / f \in C^{\infty}(M, \mathbb{R})\right\}
$$

of $\operatorname{End}_{\mathbb{C}}(\Gamma(K))$ and define on this the bracket

$$
[\hat{f}, \hat{g}]^{\varphi}=[\hat{f}, \hat{g}]-D_{\varphi\left(\Lambda^{\sharp}(d f), \Lambda^{\sharp}(d g), \cdot\right), \quad \hat{f}, \hat{g} \in A,}
$$

where $[\hat{f}, \hat{g}]=\hat{f} \circ \hat{g}-\hat{g} \circ \hat{f}$, in order to obtain a faithful representation of $\left(C^{\infty}(M, \mathbb{R}),\{\cdot, \cdot\}\right)$ on $\left(A,[\cdot, \cdot]^{\varphi}\right)$.

Proposition 4.1 The representation ${ }^{-}:\left(C^{\infty}(M, \mathbb{R}),\{\cdot, \cdot\}\right) \rightarrow\left(A,[\cdot, \cdot]^{\varphi}\right)$ is a homomorphism, i.e., for all $f, g \in C^{\infty}(M, \mathbb{R})$,

$$
\widehat{\{f, g\}}=[\hat{f}, \hat{g}]^{\varphi}
$$

if, and only if,

$$
C_{D}(d f, d g)=-2 \pi i\{f, g\} .
$$

Proof. By a simple computation, using (22) and (11), we get

$$
[\hat{f}, \hat{g}]=\hat{f} \circ \hat{g}-\hat{g} \circ \hat{f}=D_{d f} \circ D_{d g}-D_{d g} \circ D_{d f}+4 \pi i\{f, g\} .
$$

On the other hand, we have

$$
\begin{array}{rll}
\widehat{\{f, g\}} & \stackrel{(22)}{=} & D_{d\{f, g\}}+2 \pi i\{f, g\} \\
& \stackrel{(6)(10)}{=} & D_{\{d f, d g\}}-D_{\varphi\left(\Lambda^{\sharp}(d f), \Lambda^{\sharp}(d g), \cdot\right)}+2 \pi i\{f, g\} \\
& \stackrel{(13)}{=} & D_{d f} \circ D_{d g}-D_{d g} \circ D_{d f}-C_{D}(d f, d g) \\
& -D_{\varphi\left(\Lambda^{\sharp}(d f), \Lambda^{\sharp}(d g), \cdot\right)}+4 \pi i\{f, g\}-2 \pi i\{f, g\} \\
& \stackrel{(26)(23)}{=} & {[\hat{f}, \hat{g}]^{\varphi}-C_{D}(d f, d g)-2 \pi i\{f, g\} .}
\end{array}
$$

Thus, (24) holds if, and only if, (25) holds. 
Definition 4.2 We say that a twisted Poisson manifold $(M, \Lambda, \varphi)$ is prequantizable if there exists a Hermitian complex line bundle $\pi: K \rightarrow M$, the prequantization bundle, such that the operators (22) make sense on $\Gamma(K)$ and satisfy (24).

Hence, according to Proposition 4.1 and the above Definition, the prequantization problem of a twisted Poisson manifold $(M, \Lambda, \varphi)$ has a solution if, and only if, there exists a Hermitian complex line bundle $\pi: K \rightarrow M$ equipped with a contravariant derivative $D$ whose the curvature $C_{D}$ satisfies

$$
C_{D}=-2 \pi i \Lambda
$$

We see that $C_{D}$ must be purely imaginary, fact that obliges us to consider $D$ compatible with the Hermitian structure of $\pi: K \rightarrow M$.

Theorem 4.3 A twisted Poisson manifold $(M, \Lambda, \varphi)$ is prequantizable if, and only if, there exist a vector field $Z$ on $M$ and a closed 2-form $\Phi$ on $M$, which represents an integral cohomology class of $M$, such that the following relation holds on $M$ :

$$
\Lambda+\partial_{\varphi} Z=\Lambda^{\sharp}(\Phi) .
$$

Proof. We consider that $(M, \Lambda, \varphi)$ is prequantizable. Then, there exists a Hermitian complex line bundle $\pi: K \rightarrow M$ with a Hermitian contravariant derivative $D$ whose curvature $C_{D}$ verifies (27), consequently

$$
\Lambda=\frac{i}{2 \pi} C_{D} \stackrel{(14)}{=} \frac{i}{2 \pi} \Pi
$$

where $\Pi$ is the purely imaginary, $\partial_{\varphi}$-closed, bivector field on $M$ associated to $C_{D}$. On the other hand, let $\nabla$ be a Hermitian connection on $\pi: K \rightarrow M$ with curvature 2-form $\Omega$, i.e., for all $X, Y \in \Gamma(T M)$ and $s \in \Gamma(K), C_{\nabla}(X, Y)(s)=\Omega(X, Y) s$, that is purely imaginary and closed. So, $\Phi=\frac{i}{2 \pi} \Omega$ is a real closed 2-form on $M$ and represents the first real Chern class $c_{1}(K, \mathbb{R})$ of $\pi: K \rightarrow M$ which is integral, i.e., $c_{1}(K, \mathbb{R})=[\Phi]$ (see, section 3). Now, we consider the Hermitian contravariant derivative $\bar{D}$ on $\pi: K \rightarrow M$ defined by $\nabla$, i.e., for any $\alpha \in \Gamma\left(T^{*} M\right), \bar{D}_{\alpha}=\nabla_{\Lambda^{\sharp}(\alpha)}$. Let $\bar{\Pi}$ be the purely imaginary bivector field on $M$ associated to $C_{\bar{D}}$ as in (14). According to Theorem 3.6, we have $\Lambda^{\sharp}([\Phi])=\left[\frac{i}{2 \pi} \bar{\Pi}\right]^{\varphi} \stackrel{(9)}{\Leftrightarrow}\left[\Lambda^{\sharp}(\Phi)\right]^{\varphi}=\left[\frac{i}{2 \pi} \bar{\Pi}\right]^{\varphi}$. But, property (iii) of Theorem 3.4 yields $\left.[\bar{\Pi}]^{\varphi}=[\Pi]\right]^{\phi}$, which means that there exists a purely imaginary vector field $W$ on $M$ such that $\bar{\Pi}=\Pi+\partial_{\varphi} W$. Hence,

$$
\frac{i}{2 \pi} \bar{\Pi}=\frac{i}{2 \pi} \Pi+\frac{i}{2 \pi} \partial_{\varphi} W \Leftrightarrow \Lambda^{\sharp}(\Phi)=\Lambda+\partial_{\varphi} Z,
$$

where $Z=\frac{i}{2 \pi} W$.

Conversely, we assume that there exist a vector field $Z$ and a closed 2-form $\Phi$ on $(M, \Lambda, \varphi)$ such that $(28)$ is true on $M$. Then, there exists a Hermitian complex line bundle $\pi: K \rightarrow M$ over $M$ equipped with a Hermitian connection $\nabla$ having as curvature 2 -form the purely imaginary closed 2 -form $-2 \pi i \Phi$. Using $\nabla$, we define a contravariant derivative $D: \Gamma\left(T^{*} M\right) \rightarrow \operatorname{End}_{\mathbb{C}}(\Gamma(K))$ on $\pi: K \rightarrow M$ as follows: for all $\alpha \in \Gamma\left(T^{*} M\right)$ and $s \in \Gamma(K)$,

$$
D_{\alpha} s=\nabla_{\Lambda^{\sharp}(\alpha)} s+2 \pi i\langle\alpha, Z\rangle s .
$$


By a straightforward computation, we can prove that $D$ is Hermitian. Also, we have that its curvature $C_{\bar{D}}$ satisfies (27). In fact, for all $\alpha, \beta \in \Gamma\left(T^{*} M\right)$ and $s \in \Gamma(K)$,

$$
\begin{aligned}
& C_{D}(\alpha, \beta)(s) \stackrel{\stackrel{13}{=}}{=} \quad\left(D_{\alpha} \circ D_{\beta}-D_{\beta} \circ D_{\alpha}-D_{\{\alpha, \beta\}^{\varphi}}\right)(s) \\
& \stackrel{(30)}{=} \quad D_{\alpha}\left(\nabla_{\Lambda^{\sharp}(\beta)} s+2 \pi i\langle\beta, Z\rangle s\right)-D_{\beta}\left(\nabla_{\Lambda^{\sharp}(\alpha)} s+2 \pi i\langle\alpha, Z\rangle s\right) \\
& -\nabla_{\Lambda^{\sharp}\left(\{\alpha, \beta\}^{\varphi}\right)} s-2 \pi i\left\langle\{\alpha, \beta\}^{\varphi}, Z\right\rangle s \\
& \stackrel{(30)}{=} \quad \nabla_{\Lambda^{\sharp}(\alpha)}\left(\nabla_{\Lambda^{\sharp}(\beta)} s+2 \pi i\langle\beta, Z\rangle s\right)+2 \pi i\langle\alpha, Z\rangle\left(\nabla_{\Lambda^{\sharp}(\beta)} s+2 \pi i\langle\beta, Z\rangle s\right) \\
& -\nabla_{\Lambda^{\sharp}(\beta)}\left(\nabla_{\Lambda^{\sharp}(\alpha)} s+2 \pi i\langle\alpha, Z\rangle s\right)-2 \pi i\langle\beta, Z\rangle\left(\nabla_{\Lambda^{\sharp}(\alpha)} s+2 \pi i\langle\alpha, Z\rangle s\right) \\
& -\nabla_{\left[\Lambda^{\sharp}(\alpha), \Lambda^{\sharp}(\beta)\right]} s-2 \pi i\left\langle\{\alpha, \beta\}^{\varphi}, Z\right\rangle s \\
& =C_{\nabla}\left(\Lambda^{\sharp}(\alpha), \Lambda^{\sharp}(\beta)\right) s+2 \pi i\left(\Lambda^{\sharp}(\alpha)\langle\beta, Z\rangle-\Lambda^{\sharp}(\beta)\langle\alpha, Z\rangle\right. \\
& \left.-\left\langle\{\alpha, \beta\}^{\varphi}, Z\right\rangle\right) s \\
& =\quad-2 \pi i \Phi\left(\Lambda^{\sharp}(\alpha), \Lambda^{\sharp}(\beta)\right) s+2 \pi i \partial_{\varphi} Z(\alpha, \beta) s
\end{aligned}
$$

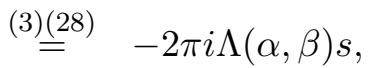

whence we conclude that $(M, \Lambda, \varphi)$ is prequantizable.

Remark 4.4 Since the first Chern class of a complex line bundle over a differentiable manifold $M$ is a complete invariant used to classify complex line bundles over $M$, i.e., there is a bijection between the isomorphism classes of complex line bundles over $M$ and the elements of $H_{d R}^{2}(M, \mathbb{Z})([8])$, we have that $K$ is not unique. Any other Hermitian complex line bundle over $M$ isomorphic to $K$ can be viewed as a prequantization bundle of $(M, \Lambda, \varphi)$.

\subsection{Examples}

1) Poisson manifolds: Let $(M, \Lambda, \varphi)$ be a twisted Poisson manifold such that $\Lambda^{\sharp}(\varphi)=0$, i.e. $(M, \Lambda)$ is a Poisson manifold. Then, the cotangent bundle $T^{*} M$ of $M$ is equipped with two different Lie algebroids structures $\left(\{\cdot, \cdot\}, \Lambda^{\sharp}\right)$ and $\left(\{\cdot, \cdot\}^{\varphi}, \Lambda^{\sharp}\right)$ whose the brackets are given, respectively, by (7) and (6). If $D$ is a contravariant derivative on an Hermitian complex line bundle $\pi: K \rightarrow M$ over $M$, then its curvatures $R_{D}$ and $C_{D}$ with respect to $\{\cdot, \cdot \cdot\}$ and $\{\cdot, \cdot\}^{\varphi}$, respectively, are related, for any $\alpha, \beta \in \Gamma\left(T^{*} M\right)$, by

$$
C_{D}(\alpha, \beta)=R_{D}(\alpha, \beta)-D_{\varphi\left(\Lambda^{\sharp}(\alpha), \Lambda^{\sharp}(\beta), \cdot\right)} .
$$

Hence, according to Definition 4.2, Proposition 4.1, and the formulæ (23) and (31), we conclude that $(M, \Lambda)$ is prequantizable as Poisson manifold ([24]) if and only if $(M, \Lambda, \varphi)$ is prequantizable as twisted Poisson manifold.

2) Twisted Poisson manifolds associated to symplectic manifolds I: Any twisted Poisson structure $(\Lambda, \varphi)$ on a $2 n$-dimensional differentiable manifold $M_{0}, n \geq 2$, constructed by a symplectic structure $\omega_{0}$ on $M_{0}$ as in Example 2 of the subsection 2.1, i.e. $\Lambda=f \Lambda_{0}$ and $\varphi=-f^{-2} \omega_{0} \wedge d f$, where $\Lambda_{0}=\Lambda_{0}^{\sharp}\left(\omega_{0}\right)$ and $f$ is an arbitrary non constant function on $M_{0}$, is not prequantizable. We will prove that the prequantization equation (28) has not solutions on $M_{0}$. We note that every vector field $Z$ on $M_{0}$ can be written as $Z=\Lambda_{0}^{\sharp}(\alpha)$ with $\alpha \in \Gamma\left(T^{*} M\right)$. Therefore,

$$
\Lambda+\partial_{\varphi} Z=\Lambda_{0}^{\sharp}\left(f \omega_{0}-f d \alpha-\alpha \wedge d f\right) .
$$


On the other hand, if there exists a closed 2-form $\Phi$ on $M_{0}$ such that, for a particular vector field $Z$ on $M_{0}, \Lambda+\partial_{\varphi} Z=\Lambda^{\sharp}(\Phi)=f^{2} \Lambda_{0}^{\sharp}(\Phi)$, then, taking into account (32) and the fact that $\Lambda_{0}^{\sharp}$ is inversible, we will must have

$$
f^{2} \Phi=f \omega_{0}-f d \alpha-\alpha \wedge d f \Leftrightarrow \Phi=f^{-1} \omega_{0}-f^{-1} d \alpha-f^{-2} \alpha \wedge d f .
$$

But, in this case, $d \Phi=-f^{-2} \omega_{0} \wedge d f=\varphi \neq 0$, for any non constant function $f$ on $M_{0}$. Thus, $\left(M_{0}, \Lambda, \varphi\right)$ is not prequantizable.

3) Twisted Poisson manifolds associated to symplectic manifolds $I I$ : Let $(M, \Lambda, \varphi)$ be a twisted Poisson manifold constructed by a symplectic manifold $\left(M_{0}, \omega_{0}\right)$ as in Example 3 of the subsection 2.1 , i.e., $M=M_{0} \times \mathbb{R}$,

$$
\Lambda=e^{t}\left(\Lambda_{0}+\Lambda_{0}^{\sharp}(d f) \wedge \frac{\partial}{\partial t}\right) \quad \text { and } \quad \varphi=-e^{-t} \omega_{0} \wedge d t
$$

$t$ being the canonical coordinate on $\mathbb{R}$ and $f \in C^{\infty}\left(M_{0}, \mathbb{R}\right)$. We assume that the symplectic structure $\omega_{0}$ is of the particular type $\omega_{0}=d \alpha_{0}-\alpha_{0} \wedge d f$, where $\alpha_{0}$ is a convenient 1-form on $M_{0}$, i.e., $\alpha_{0}$ is a 1-form on $M_{0}$ such that $\omega_{0}=d \alpha_{0}-\alpha_{0} \wedge d f$ is nondegenerate and $d \omega_{0}=-d \alpha_{0} \wedge d f=0$. Then, $(M, \Lambda, \varphi)$ is prequantizable. Effectively, if we take $Z=\partial / \partial t$ and $\Phi=d\left(e^{-t} \alpha_{0}\right)$, which represents the integral cohomology class $[0] \in H_{d R}^{2}(M, \mathbb{R})$ of $M$, after a simple computation we obtain that (28) holds on $M$.

4) Exact twisted Poisson manifolds: Let $(M, \Lambda, \varphi)$ be an exact twisted Poisson manifold, namely, there exists a vector field $X$ on $M$ such that $\Lambda=\partial_{\varphi} X$, fact that is equivalent to $[\Lambda]^{\varphi}=[0]^{\varphi} \in H_{L-t P}^{2}(M)$. Then, $(M, \Lambda, \varphi)$ is prequantizable. The vector field $Z=-X$ and the 2 -form $\Phi=0$ satisfy the prequantization condition (28). The trivial complex line bundle $\pi: M \times \mathbb{C} \rightarrow M$, whose space of global cross sections $\Gamma(M \times \mathbb{C})$ is equal to the set $C^{\infty}(M, \mathbb{C})$, equipped with the usual Hermitian metric $h$, i.e., for any $s_{1}, s_{2} \in C^{\infty}(M, \mathbb{C}), h\left(s_{1}, s_{2}\right)=s_{1} \bar{s}_{2}$, and the compatible with $h$ contravariant derivative $D$ given, for any $\alpha \in \Gamma\left(T^{*} M\right)$ and $s \in C^{\infty}(M, \mathbb{C})$, by $D_{\alpha} s=\Lambda^{\sharp}(\alpha) s$, is a prequantization bundle of $(M, \Lambda, \varphi)$.

5) Twisted Poisson structures induced by twisted Jacobi manifolds: Let $\left(M_{0}, \Lambda_{0}, d \omega_{0}\right)$ be the twisted Poisson manifold constructed by a twisted Jacobi manifold $\left(M, \Lambda, E, \varpi^{*} \omega_{0}\right)$ in Example 4 of the subsection 2.1. Let $\eta$ be the 1-form along $M_{0}$ that verifies $i(E) \eta=1$ and $i(X) \eta=0$, for any vector field $X$ on $M$ tangent to $M_{0}$. By integration along the integral curves of $E$ and by restriction, if necessary, of the tubular neighbourhood $U$ of $M_{0}$ in $M$, we can construct a function $h$ on $M$ such that $\left.h\right|_{M_{0}}=0$ and $i(E) d h=1$, hence $\left.d h\right|_{M_{0}}=\eta$. Let $X_{h}=\Lambda^{\sharp}(d h)+h E$ be the Hamiltonian vector field of $h$ with respect the twisted Jacobi structure $(\Lambda, E)$ on $M$. Since $[E, \Lambda](d h, \cdot)=0$, we have $\left[E, X_{h}\right]=E$, whence we conclude that $X_{h}$ is projectable along the integral curves of $E$ onto $M_{0}$. Let $Z_{0}$ be its projection, i.e. $Z_{0}=\varpi_{*} X_{h}=\varpi_{*}\left(\Lambda^{\sharp}(d h)\right)=\Lambda^{\sharp}(\eta)$. The differential operator of first order $X_{h}-1$ verifies (see in [15] Propositions 3.1 and 3.5), for any $f, g \in C^{\infty}(M, \mathbb{R})$, the relation

$$
\begin{aligned}
\left(X_{h}-1\right)\{f, g\}= & \{h,\{f, g\}\} \\
= & \{\{h, f\}, g\}+\{f,\{h, g\}\} \\
& +\Lambda^{\sharp}\left(\varpi^{*} d \omega_{0}\right)(d h, d f, d g)+\Lambda^{\sharp}\left(\varpi^{*} \omega_{0}\right) \wedge E(d h, d f, d g) .
\end{aligned}
$$

By projection, we obtain that the first order differential operator $Z_{0}-1$ verifies, for any $f_{0}, g_{0} \in C^{\infty}\left(M_{0}, \mathbb{R}\right)$,

$$
\begin{aligned}
\left(Z_{0}-1\right)\left\{f_{0}, g_{0}\right\}= & \left\{\left(Z_{0}-1\right) f_{0}, g_{0}\right\}+\left\{f_{0},\left(Z_{0}-1\right) g_{0}\right\} \\
& -d \omega_{0}\left(Z_{0}, \Lambda_{0}^{\sharp}\left(d f_{0}\right), \Lambda_{0}^{\sharp}\left(d g_{0}\right)\right)+\omega_{0}\left(\Lambda_{0}^{\sharp}\left(d f_{0}\right), \Lambda_{0}^{\sharp}\left(d g_{0}\right)\right) .
\end{aligned}
$$


From the above relation, after a simple computation, we get

$$
\Lambda_{0}+\partial_{d \omega_{0}}\left(-Z_{0}\right)=\Lambda_{0}^{\sharp}\left(\omega_{0}\right) .
$$

If $\omega_{0}$ is closed and represents an integral cohomology class of $M_{0}$, then, the last equation means that the induced twisted Poisson structure $\left(\Lambda_{0}, d \omega_{0}\right)=\left(\Lambda_{0}, 0\right)$ is a prequantizable Poisson structure on $M_{0}$. (For more details, see [17].)

6) A r-matrix type twisted Poisson structure: We consider the twisted Poisson structure of the Example 4.8 in [10] (see, also Example 5 in [9]). Let $\mathcal{G}$ be the subalgebra of the Lie algebra of $G L(3, \mathbb{R})$ spanned by $\left\{e_{i j} / 1 \leq i \leq 2,1 \leq j \leq 3\right\}$. We denote by $\left\{e_{i j}^{*} / 1 \leq i \leq 2,1 \leq j \leq 3\right\}$ the dual basis of its dual space $\mathcal{G}^{*}$. The pair $(r, \varphi)$, where

$$
r=e_{11} \wedge e_{22}+e_{13} \wedge e_{23} \quad \text { and } \varphi=-\left(e_{11}^{*}+e_{22}^{*}\right) \wedge e_{13}^{*} \wedge e_{23}^{*},
$$

defines a twisted Poisson structure on $\mathcal{G}$. It is easy to check that $\varphi$ is closed and $\frac{1}{2}[r, r]=r^{\sharp}(\varphi)$. We will show that $(r, \varphi)$ is not prequantizable on $\mathcal{G}$. After a simple, but long, computation, we prove that the space of closed 2 -forms of $\mathcal{G}$ is spanned by $\left\{\left(e_{11}^{*}-e_{22}^{*}\right) \wedge e_{12}^{*},\left(e_{11}^{*}-e_{22}^{*}\right) \wedge e_{21}^{*}, e_{12}^{*} \wedge e_{21}^{*}\right\}$ and that, for any such form $\Phi$ of $\mathcal{G}, r^{\sharp}(\Phi)=0$. On the other hand, for any vector $Z=\sum_{i, j} \lambda_{i j} e_{i j}, \lambda_{i j} \in \mathbb{R}$, of $\mathcal{G}$, we have

$$
\begin{aligned}
r+\partial_{\varphi} Z= & -\lambda_{12} e_{11} \wedge e_{12}-\lambda_{13} e_{11} \wedge e_{13}+\lambda_{21} e_{11} \wedge e_{21}+e_{11} \wedge e_{22}+\lambda_{12} e_{12} \wedge e_{22} \\
& +\left(1-\lambda_{12}+\lambda_{21}\right) e_{13} \wedge e_{23}-\lambda_{21} e_{21} \wedge e_{22}+\lambda_{23} e_{22} \wedge e_{23} \neq 0 .
\end{aligned}
$$

Hence, we conclude that the prequantization equation (28) has not a solution $(Z, \Phi)$ on $\mathcal{G}$.

\section{Quantization}

The second step of the geometric quantization of a twisted Poisson manifold $(M, \Lambda, \varphi)$ is the construction of a Hilbert space out of its prequantization space $\Gamma(K)$ on which a convenient $\varphi$-twisted Lie subalgebra of the $\varphi$-twisted Lie algebra $\left(C^{\infty}(M, \mathbb{R}),\{\cdot, \cdot\}\right)$ will be represented irreducibly. For this reason, we introduce the notion of polarization ([20], [29]) of $(M, \Lambda, \varphi)$ as follows.

We consider the complexification $T^{*} M \otimes \mathbb{C}$ of the cotangent bundle $T^{*} M$ of $M$ and we endow the space of its cross sections $\Gamma\left(T^{*} M \otimes \mathbb{C}\right)$ with the natural extension of the bracket (6), also denoted by $\{\cdot, \cdot\}^{\varphi}$. Then, $\left(T^{*} M \otimes \mathbb{C},\{\cdot, \cdot\}^{\varphi}, \Lambda^{\sharp}\right)$, where $\Lambda^{\sharp}: T^{*} M \otimes \mathbb{C} \rightarrow$ $T M \otimes \mathbb{C}$ is the natural extension to $T^{*} M \otimes \mathbb{C}$ of the vector bundle map given by (2), is a complex Lie algebroid over $M$, in the sense of [3], and $\left(\Gamma\left(T^{*} M \otimes \mathbb{C}\right),\{\cdot, \cdot\}^{\varphi}\right)$ is a complex Lie algebra. We define a polarization of $(M, \Lambda, \varphi)$ to be a complex Lie subalgebra $\mathcal{P}$ of $\left(\Gamma\left(T^{*} M \otimes \mathbb{C}\right),\{\cdot, \cdot\}^{\varphi}\right)$ such that, for all $\alpha, \beta \in \mathcal{P}$,

$$
\Lambda(\alpha, \beta)=0 .
$$

When $\mathcal{P}$ is fixed, we set

$$
P(\mathcal{P})=\left\{f \in C^{\infty}(M, \mathbb{R}) /\{d f, \alpha\}^{\varphi} \in \mathcal{P}, \quad \text { for all } \alpha \in \mathcal{P}\right\}
$$

and we consider the subset $\widetilde{P(\mathcal{P})}$ of $P(\mathcal{P}) \times P(\mathcal{P})$ given by

$$
\begin{aligned}
\widetilde{P(\mathcal{P})}=\{( & f, g) \in P(\mathcal{P}) \times P(\mathcal{P}) \backslash \Delta(P(\mathcal{P}) \times P(\mathcal{P})) / \\
& \left.\left\{\varphi\left(\Lambda^{\sharp}(d f), \Lambda^{\sharp}(d g), \cdot\right), \alpha\right\}^{\varphi} \in \mathcal{P}, \quad \text { for all } \alpha \in \mathcal{P}\right\},
\end{aligned}
$$


where $\Delta(P(\mathcal{P}) \times P(\mathcal{P}))$ denotes the diagonal of $P(\mathcal{P}) \times P(\mathcal{P})$. Clearly, $\widetilde{P(\mathcal{P})}$ is symmetric with respect to $\Delta(P(\mathcal{P}) \times P(\mathcal{P}))$. If $\mathcal{Q}(\mathcal{P})$ is the projection of $\widehat{P(\mathcal{P})}$ on $P(\mathcal{P})$, we have that $(\mathcal{Q}(\mathcal{P}),\{\cdot, \cdot\})$ is a $\varphi$-twisted Lie subalgebra of $\left(C^{\infty}(M, \mathbb{R}),\{\cdot, \cdot\}\right)$ which will be called the subalgebra of the straightforwardly quantizable observables of $(M, \Lambda, \varphi)$. Obviously, if $\varphi=0$, from the above definitions we obtain those given in [24] for Poisson manifolds.

Now, in order to build a Hilbert space out of $\Gamma(K)$ on which the quantum operators corresponding to the elements of $\mathcal{Q}(\mathcal{P})$ act, we apply a classical method in geometric quantization using the line bundle of complex half-densities of $M$.

Let $\mathcal{D}$ be the half-density bundle associated to $T M$. It is well known ([2], [22], [26]) that its cross sections $\varrho$, called half-densities of $M$, are complex valued maps defined on the set $\mathcal{B}(T M)$ of basis of $\Gamma(T M)$ such that, for any $x \in M, e_{x} \in \mathcal{B}\left(T_{x} M\right)$ and $A_{x} \in G L\left(T_{x} M\right)$,

$$
\varrho_{x}\left(e_{x} A_{x}\right)=\varrho_{x}\left(e_{x}\right)\left|\operatorname{det} A_{x}\right|^{1 / 2} .
$$

Since $G L\left(T_{x} M\right)$ acts transitively on $\mathcal{B}\left(T_{x} M\right), \varrho_{x}$ is determined by its value on a single basis of $\Gamma\left(T_{x} M\right)$. As a result, we have that $\mathcal{D}$ is a complex line bundle over $M$ which is defined by the transition functions that are the square roots of the absolute values of the Jacobians of the coordinate transformations $\tilde{x}_{i}=\tilde{x}_{i}\left(x_{j}\right)$, i.e. $\left|\partial x_{j} / \partial \tilde{x}_{i}\right|^{1 / 2}$. The Lie derivatives $\mathcal{L}$ of $\varrho$ are defined as for tensors fields on $M$, (see, [26]).

We assume that $(M, \Lambda, \varphi)$ is a prequantizable twisted Poisson manifold. Let $\pi$ : $K \rightarrow M$ be its prequantization bundle, $h$ the Hermitian metric on $\pi: K \rightarrow M$ and $D$ a compatible with $h$ contravariant derivative on $\pi: K \rightarrow M$ whose curvature $C_{D}$ verifies (27). Using the properties (10), (11) of $D$ and those of $\mathcal{L}$, we can extend $D$ to a mapping, also denoted by $D$,

$$
D: \Gamma\left(T^{*} M \otimes \mathbb{C}\right) \rightarrow \operatorname{End}_{\mathbb{C}}(\Gamma(K \otimes \mathcal{D}))
$$

by putting, for any $\alpha \in \Gamma\left(T^{*} M \otimes \mathbb{C}\right)$ and $s \otimes \varrho \in \Gamma(K \otimes \mathcal{D})$,

$$
D_{\alpha}(s \otimes \varrho)=D_{\alpha} s \otimes \varrho+s \otimes \mathcal{L}_{\Lambda^{\sharp}(\alpha)} \varrho .
$$

Therefore, the representation ${ }^{\wedge}:\left(C^{\infty}(M, \mathbb{R}),\{\cdot, \cdot\}\right) \rightarrow \operatorname{End}_{\mathbb{C}}(\Gamma(K))$ given by $(22)$ can be extended to a representation of $\left(C^{\infty}(M, \mathbb{R}),\{\cdot, \cdot\}\right)$ on $\Gamma(K \otimes \mathcal{D})$, also denoted by $\widehat{\text {, }}$ by setting, for all $f \in C^{\infty}(M, \mathbb{R})$ and $s \otimes \varrho \in \Gamma(K \otimes \mathcal{D})$,

$$
\hat{f}(s \otimes \varrho)=D_{d f}(s \otimes \varrho)+2 \pi i f(s \otimes \varrho) .
$$

Because of (33), (34) can be written as

$$
\hat{f}(s \otimes \varrho)=(\hat{f}(s)) \otimes \varrho+s \otimes \mathcal{L}_{\Lambda^{\sharp}(d f)} \varrho .
$$

Thus, taking into account (35), (24), (6), the property of the anchor map $\Lambda^{\sharp}$ and that of the Lie derivative, we can easily check that the prequantization condition (24) remains true, i.e., for any $f, g \in C^{\infty}(M, \mathbb{R})$ and $s \otimes \varrho \in \Gamma(K \otimes \mathcal{D})$,

$$
\widehat{\{f, g\}}(s \otimes \varrho)=[\hat{f}, \hat{g}]^{\varphi}(s \otimes \varrho) .
$$

Furthermore, applying (34), (33), (13) and (27), we deduce that

$$
D_{\alpha}(\hat{f}(s \otimes \varrho))=\hat{f}\left(D_{\alpha}(s \otimes \varrho)\right)-D_{\{d f, \alpha\} \varphi}(s \otimes \varrho),
$$


for all $\alpha \in \Gamma\left(T^{*} M \otimes \mathbb{C}\right), f \in C^{\infty}(M, \mathbb{R})$ and $s \otimes \varrho \in \Gamma(K \otimes \mathcal{D})$.

Now, for a fixed polarization $\mathcal{P}$ of $(M, \Lambda, \varphi)$, we consider the subset $\mathcal{H}_{0}$ of $\Gamma(K \otimes \mathcal{D})$ given by

$$
\mathcal{H}_{0}=\left\{s \otimes \varrho \in \Gamma(K \otimes \mathcal{D}) / D_{\alpha}(s \otimes \varrho)=0, \quad \text { for all } \alpha \in \mathcal{P}\right\},
$$

and we assume that $\mathcal{H}_{0} \neq\{0\}$, which is a Bohr-Sommerfeld type condition (see, [20]). We have that, for any $f \in \mathcal{Q}(\mathcal{P})$ and $s \otimes \varrho \in \mathcal{H}_{0}, \hat{f}(s \otimes \varrho) \in \mathcal{H}_{0}$. In fact, for every $\alpha \in \mathcal{P},\{d f, \alpha\}^{\varphi} \in \mathcal{P}$ and $D_{\alpha}(s \otimes \varrho)=0$. Hence, according to (36), we get

$$
D_{\alpha}(\hat{f}(s \otimes \varrho))=\hat{f}\left(D_{\alpha}(s \otimes \varrho)\right)-D_{\{d f, \alpha\}}(s \otimes \varrho)=\hat{f}(0)-0=0,
$$

which means that $\hat{f}(s \otimes \varrho) \in \mathcal{H}_{0}$. Consequently, $\left.\hat{f}\right|_{\mathcal{H}_{0}}: \mathcal{H}_{0} \rightarrow \mathcal{H}_{0}$ is well defined for any $f \in \mathcal{Q}(\mathcal{P})$. Thus, $\mathcal{H}_{0}$ can be viewed as a quantization space for $\mathcal{Q}(\mathcal{P})$.

Next, we distinguish the following cases.

If $M$ is compact, then, $\mathcal{H}_{0}$ equipped with the inner product

$$
\left\langle s_{1} \otimes \varrho_{1}, s_{2} \otimes \varrho_{2}\right\rangle=\int_{M} h\left(s_{1}, s_{2}\right) \varrho_{1} \bar{\varrho}_{2},
$$

$h$ being the Hermitian metric on $\pi: K \rightarrow M$ and bar denoting the complex conjugation, is a pre-Hilbert space. Moreover, the operators $\hat{f}$ defined by (34) or (35) are antiHermitian with respect to (38). This results as follows:

$$
\begin{aligned}
& \left\langle\hat{f}\left(s_{1} \otimes \varrho_{1}\right), s_{2} \otimes \varrho_{2}\right\rangle+\left\langle s_{1} \otimes \varrho_{1}, \hat{f}\left(s_{2} \otimes \varrho_{2}\right)\right\rangle \stackrel{(35)}{=} \\
& \left\langle\left(\hat{f} s_{1}\right) \otimes \varrho_{1}+s_{1} \otimes \mathcal{L}_{\Lambda^{\sharp}(d f)} \varrho_{1}, s_{2} \otimes \varrho_{2}\right\rangle+\left\langle s_{1} \otimes \varrho_{1},\left(\hat{f} s_{2}\right) \otimes \varrho_{2}+s_{2} \otimes \mathcal{L}_{\Lambda^{\sharp}(d f)} \varrho_{2}\right\rangle \stackrel{(38)}{=} \\
& \int_{M}\left(\left(h\left(\hat{f} s_{1}, s_{2}\right)+h\left(s_{1}, \hat{f} s_{2}\right)\right) \varrho_{1} \bar{\varrho}_{2}+h\left(s_{1}, s_{2}\right)\left(\left(\mathcal{L}_{\Lambda^{\sharp}(d f)} \varrho_{1}\right) \bar{\varrho}_{2}+\varrho_{1}\left(\mathcal{L}_{\Lambda^{\sharp}(d f)} \bar{\varrho}_{2}\right)\right)\right) \stackrel{(12)}{=} \\
& \int_{M}\left(\Lambda^{\sharp}(d f)\left(h\left(s_{1}, s_{2}\right)\right) \varrho_{1} \bar{\varrho}_{2}+h\left(s_{1}, s_{2}\right)\left(\left(\mathcal{L}_{\Lambda^{\sharp}(d f)} \varrho_{1}\right) \varrho_{2}+\varrho_{1}\left(\mathcal{L}_{\Lambda^{\sharp}(d f)} \bar{\varrho}_{2}\right)\right)\right)= \\
& \int_{M} \mathcal{L}_{\Lambda^{\sharp}(d f)}\left(h\left(s_{1}, s_{2}\right) \varrho_{1} \bar{\varrho}_{2}\right)=0,
\end{aligned}
$$

where the last equality is true because of the density version of Stokes' Theorem ([23], [22]). If we require the quantization space for $\mathcal{Q}(\mathcal{P})$ is a Hilbert space, we take the completion $\mathcal{H}$ of $\mathcal{H}_{0}$. In order to obtain Hermitian operators on $\mathcal{H}$, we prolong $\hat{f}$ on $\mathcal{H}$ so that the obtained operators are anti-Hermitian and then we multiple these by $i$. Then, condition (24) is true up to the constant factor $i$.

If $M$ is not compact, we consider the subalgebra $\mathcal{P}_{0}$ of $\left(\Gamma\left(T^{*} M\right),\{\cdot, \cdot\}^{\varphi}\right)$ whose complexification is $\mathcal{P} \cap \overline{\mathcal{P}}$ (so, for all $\alpha, \beta \in \mathcal{P}_{0}, \Lambda(\alpha, \beta)=0$ ) and we postulate $\Lambda^{\sharp}\left(\mathcal{P}_{0}\right)$ to defines a regular foliation $\mathcal{F}$ of $M$ whose the leaf space $N=M / \mathcal{F}$ is a Hausdorff manifold. We can easily show that, for any $f \in \mathcal{Q}(\mathcal{P})$ and $\alpha \in \mathcal{P}_{0},\{d f, \alpha\}^{\varphi} \in \mathcal{P}_{0}$. Therefore, for any $f \in \mathcal{Q}(\mathcal{P})$, the Hamiltonian vector field $\Lambda^{\sharp}(d f)$ is projectable with respect to $\Lambda^{\sharp}\left(\mathcal{P}_{0}\right)$ onto $N$ (we have, for all $\alpha \in \mathcal{P}_{0},\left[\Lambda^{\sharp}(d f), \Lambda^{\sharp}(\alpha)\right]=\Lambda^{\sharp}\left(\{d f, \alpha\}^{\varphi}\right) \in$ $\left.\Lambda^{\sharp}\left(\mathcal{P}_{0}\right)\right)$. Also, if $\varpi: M \rightarrow N$ denotes the canonical projection of $M$ onto $N$, we have

$$
\begin{aligned}
\hat{f}\left(s \otimes \varpi^{*} \varrho_{N}\right) & =(\hat{f} s) \otimes \varpi^{*} \varrho_{N}+s \otimes \mathcal{L}_{\Lambda^{\sharp}(d f)}\left(\varpi^{*} \varrho_{N}\right) \\
& =(\hat{f} s) \otimes \varpi^{*} \varrho_{N}+s \otimes \varpi^{*}\left(\mathcal{L}_{\varpi *}\left(\Lambda^{\sharp}(d f)\right) \varrho_{N}\right),
\end{aligned}
$$

for all $s \in \Gamma(K)$ and $\varrho_{N}$ a complex half-density of $N$. The last equality permits us, instead of using arbitrary half-densities of $M$ for the construction of $\mathcal{H}_{0}$, to use $\mathcal{F}$ transversal half-densities of $M$ that are the pull-back under $\varpi$ of half-densities of $N$. 
Then, for any $\alpha \in \mathcal{P}_{0}$ and $\varrho_{N}$ complex half-density of $N, \mathcal{L}_{\Lambda^{\sharp}(\alpha)}\left(\varpi^{*} \varrho_{N}\right)=0$. Using this fact, (12), (33) and (37), we have that, for all $s_{1} \otimes \varpi^{*} \varrho_{1_{N}}, s_{2} \otimes \varpi^{*} \varrho_{2_{N}} \in \mathcal{H}_{0}$ and $\alpha \in \mathcal{P}_{0}$,

$$
\mathcal{L}_{\Lambda^{\sharp}(\alpha)}\left(h\left(s_{1}, s_{2}\right) \varpi^{*} \varrho_{1_{N}} \varpi^{*} \bar{\varrho}_{2_{N}}\right)=0,
$$

which means that $h\left(s_{1}, s_{2}\right) \varpi^{*} \varrho_{1_{N}} \varpi^{*} \bar{\varrho}_{2_{N}}$ can be projected to a complex 1-density $\delta_{N}$ of $N$ (the multiplication of two half-densities yields a 1-density). Hence, $\mathcal{H}_{0}$ can be replaced by its subspace $\mathcal{H}_{0}^{c}$ formed by the sections that are projectable to $N$ and whose projection has as support a compact subset of $N$. In general, we may expect that $\mathcal{H}_{0}^{c} \neq\{0\}$. In this case, $\mathcal{H}_{0}^{c}$ endowed with the inner product

$$
\left\langle s_{1} \otimes \varpi^{*} \varrho_{1_{N}}, s_{2} \otimes \varpi^{*} \varrho_{2_{N}}\right\rangle=\int_{N} \delta_{N}
$$

is a pre-Hilbert space. Furthermore, working as in (39), we prove that, for any $f \in$ $\mathcal{Q}(\mathcal{P})$, the corresponding operator $\hat{f}$ verifies

$$
\left\langle\hat{f}\left(s_{1} \otimes \varpi^{*} \varrho_{1_{N}}\right), s_{2} \otimes \varpi^{*} \varrho_{2_{N}}\right\rangle+\left\langle s_{1} \otimes \varpi^{*} \varrho_{1_{N}}, \hat{f}\left(s_{2} \otimes \varpi^{*} \varrho_{2_{N}}\right)\right\rangle=\int_{N} \mathcal{L}_{\Lambda^{\sharp}(d f)} \delta_{N}=0,
$$

whence we deduce the anti-Hermitian character of $\hat{f}$. In order that the quantization space of $\mathcal{Q}(\mathcal{P})$ be a Hilbert space and in order to obtain Hermitian operators on this space, we proceed as in the compact case.

\section{$5.1 \quad$ Example}

Below, we will study the quantization of the prequantizable twisted Poisson manifold $(M, \Lambda, \varphi)$ presented in Example 3 of paragraph 4.1.

We have $(M, \Lambda, \varphi)=\left(M_{0} \times \mathbb{R}, e^{t}\left(\Lambda_{0}+\Lambda_{0}^{\sharp}(d f) \wedge \frac{\partial}{\partial t}\right),-e^{-t} \omega_{0} \wedge d t\right)$, where $\left(M_{0}, \omega_{0}\right)=$ $\left(M_{0}, d \alpha_{0}-\alpha_{0} \wedge d f\right)$ is a symplectic manifold, with $\alpha_{0} \in \Gamma\left(T^{*} M_{0}\right)$ and $f \in C^{\infty}(M, \mathbb{R})$, $\Lambda_{0}=\Lambda_{0}^{\sharp}\left(\omega_{0}\right)$ and $t$ is the canonical coordinate on $\mathbb{R}$. As we have seen, a solution of (28) is $(Z, \Phi)=\left(\partial / \partial t, d\left(e^{-t} \alpha_{0}\right)\right)$, therefore, the prequantization bundle of $(M, \Lambda, \varphi)$ is the trivial complex line bundle $\pi: M \times \mathbb{C} \rightarrow M$ equipped with the usual Hermitian metric $h$ and the Hermitian contravariant derivative $D$ defined, for any $\alpha \in \Gamma\left(T^{*} M\right)$ and $s \in \Gamma(M \times \mathbb{C})=C^{\infty}(M, \mathbb{C})$, by

$$
D_{\alpha} s=\Lambda^{\sharp}(\alpha) s .
$$

We take $M_{0}=\mathbb{R}^{2 n}, n \geq 2$. Let $\left(x_{1}, x_{2}, \ldots, x_{2 n}\right)$ be a local coordinates system of $M_{0}$ in which $\omega_{0}=d \alpha_{0}-\alpha_{0} \wedge d f$ has the Darboux's expression, i.e.,

$$
\omega_{0}=\sum_{k=1}^{n} d x_{2 k-1} \wedge d x_{2 k}
$$

Hence,

$$
\Lambda=e^{t}\left(\sum_{k=1}^{n} \frac{\partial}{\partial x_{2 k-1}} \wedge \frac{\partial}{\partial x_{2 k}}+\sum_{k=1}^{n}\left(\frac{\partial f}{\partial x_{2 k-1}} \frac{\partial}{\partial x_{2 k}}-\frac{\partial f}{\partial x_{2 k}} \frac{\partial}{\partial x_{2 k-1}}\right) \wedge \frac{\partial}{\partial t}\right)
$$

and

$$
\varphi=-e^{-t}\left(\sum_{k=1}^{n} d x_{2 k-1} \wedge d x_{2 k} \wedge d t\right)
$$


Using the identifications $M=\mathbb{R}^{2 n} \times \mathbb{R} \cong \mathbb{C}^{n} \times \mathbb{R}, z_{k}=x_{2 k-1}+i x_{2 k}$ and $\bar{z}_{k}=$ $x_{2 k-1}-i x_{2 k}, k=1, \ldots, n$, which give us $d x_{2 k-1}=\frac{1}{2}\left(d z_{k}+d \bar{z}_{k}\right), d x_{2 k}=-\frac{i}{2}\left(d z_{k}-d \bar{z}_{k}\right)$, $\frac{\partial}{\partial x_{2 k-1}}=\frac{\partial}{\partial z_{k}}+\frac{\partial}{\partial \bar{z}_{k}}$ and $\frac{\partial}{\partial x_{2 k}}=i\left(\frac{\partial}{\partial z_{k}}-\frac{\partial}{\partial \bar{z}_{k}}\right)$, we obtain that, in the complex coordinates $\left(z_{1}, \ldots, z_{n}, t\right)$ of $M$, the pair $(\Lambda, \varphi)$ is written as follows:

$$
\begin{gathered}
\Lambda=-2 i e^{t}\left(\sum_{k=1}^{n} \frac{\partial}{\partial z_{k}} \wedge \frac{\partial}{\partial \bar{z}_{k}}+\sum_{k=1}^{n}\left(\frac{\partial f}{\partial z_{k}} \frac{\partial}{\partial \bar{z}_{k}}-\frac{\partial f}{\partial \bar{z}_{k}} \frac{\partial}{\partial z_{k}}\right) \wedge \frac{\partial}{\partial t}\right), \\
\varphi=-\frac{i}{2} e^{-t}\left(\sum_{k=1}^{n} d z_{k} \wedge d \bar{z}_{k} \wedge d t\right) .
\end{gathered}
$$

We observe that a convenient polarization of $(M, \Lambda, \varphi)$ is $\mathcal{P}=\operatorname{span}\left\{d z_{1}, \ldots, d z_{n}\right\}$. Then, the set $P(\mathcal{P})$ consists of the functions $g \in C^{\infty}(M, \mathbb{R})$ for that $\left\{d g, d z_{k}\right\}^{\varphi} \in \mathcal{P}$, for any $k=1, \ldots, n$. After a computation, we get that the coefficient of $d t$ in $\left\{d g, d z_{k}\right\}^{\varphi}$ is annihilated. Thus, $\left\{d g, d z_{k}\right\}^{\varphi} \in \mathcal{P}$ if, and only if, its coefficients of $d \bar{z}_{l}, l=1, \ldots, n$, are annihilated, i.e.,

$$
\frac{\partial}{\partial \bar{z}_{l}}\left(-\frac{\partial g}{\partial \bar{z}_{k}}+\frac{\partial f}{\partial \bar{z}_{k}} \frac{\partial g}{\partial t}\right)+\frac{\partial g}{\partial \bar{z}_{l}} \frac{\partial f}{\partial \bar{z}_{k}}-\frac{\partial f}{\partial \bar{z}_{k}} \frac{\partial f}{\partial \bar{z}_{l}} \frac{\partial g}{\partial t}=0, \quad \forall l=1, \ldots, n
$$

Now, we consider the set $\widetilde{P(\mathcal{P})} \subset P(\mathcal{P}) \times P(\mathcal{P})$ of the pairs $\left(g_{1}, g_{2}\right)$ of different solutions of the system (42) for that $\left\{\varphi\left(\Lambda^{\sharp}\left(d g_{1}\right), \Lambda^{\sharp}\left(d g_{2}\right), \cdot\right), d z_{k}\right\}^{\varphi} \in \mathcal{P}$, for any $k=1, \ldots, n$, and we take its projection $\mathcal{Q}(\mathcal{P})$ on $P(\mathcal{P})$. The set $\mathcal{Q}(\mathcal{P})$ is the one of straightforwardly quantizable observables of $(M, \Lambda, \varphi)$. We note that a solution of (42) is $g_{1}=f+t$. Since $\Lambda^{\sharp}\left(d g_{1}\right)=0$,

$$
\left\{\varphi\left(\Lambda^{\sharp}\left(d g_{1}\right), \Lambda^{\sharp}\left(d g_{2}\right), \cdot\right), d z_{k}\right\}^{\varphi}=\left\{\varphi\left(0, \Lambda^{\sharp}\left(d g_{2}\right), \cdot\right), d z_{k}\right\}^{\varphi}=\left\{0, d z_{k}\right\}^{\varphi}=0 \in \mathcal{P},
$$

for any other $g_{2} \in P(\mathcal{P})$ and any $d z_{k}, k=1, \ldots, n$. So, $f+t \in \mathcal{Q}(\mathcal{P})$.

Next, we have to determine the corresponding quantization space $\mathcal{H}_{0}$ for $\mathcal{Q}(\mathcal{P})$. The bundle $\mathcal{D}$ of complex half-densities over $M=\mathbb{C}^{n} \times \mathbb{R}$ is also trivial and it has a basis that can be written formally as $\beta=|v|^{1 / 2}$, where

$$
v=d x_{1} \wedge \ldots \wedge d x_{2 n} \wedge d t=\left(\frac{i}{2}\right)^{n} d z_{1} \wedge \ldots \wedge d z_{n} \wedge d \bar{z}_{1} \wedge \ldots \wedge d \bar{z}_{n} \wedge d t .
$$

Hence, taking 1 as the unitary basis of $K=M \times \mathbb{C}$, we have that any section $s \otimes \varrho$ of $K \otimes \mathcal{D}$ can be written as $s \otimes \varrho=1 \otimes(\chi \beta)$, where $\chi \in C^{\infty}(M, \mathbb{C})$. Let $D$ be the extension (33) of the Hermitian contravariant derivative on $\pi: K \rightarrow M$ given by (41). Then, according to the formula $\mathcal{L}_{X} \beta=\frac{1}{2}(\operatorname{div} X) \beta$ presented in [23] (see, also [26]), we get

$$
D_{d z_{k}}(1 \otimes(\chi \beta))=1 \otimes \mathcal{L}_{\Lambda^{\sharp}\left(d z_{k}\right)}(\chi \beta)=1 \otimes\left(\mathcal{L}_{\Lambda^{\sharp}\left(d z_{k}\right)} \chi+\frac{\chi}{2} \operatorname{div} \Lambda^{\sharp}\left(d z_{k}\right)\right) \beta=0
$$

if, and only if,

$$
\mathcal{L}_{\Lambda^{\sharp}\left(d z_{k}\right)} \chi+\frac{\chi}{2} \operatorname{div} \Lambda^{\sharp}\left(d z_{k}\right)=0 .
$$

But,

$$
\Lambda^{\sharp}\left(d z_{k}\right)=-2 i e^{t}\left(\frac{\partial}{\partial \bar{z}_{k}}-\frac{\partial f}{\partial \bar{z}_{k}} \frac{\partial}{\partial t}\right) \quad \text { and } \quad \operatorname{div} \Lambda^{\sharp}\left(d z_{k}\right)=2 i e^{t} \frac{\partial f}{\partial \bar{z}_{k}} .
$$

Thus, (43) is equivalent to

$$
-\frac{\partial \chi}{\partial \bar{z}_{k}}+\frac{\partial f}{\partial \bar{z}_{k}} \frac{\partial \chi}{\partial t}+\frac{\chi}{2} \frac{\partial f}{\partial \bar{z}_{k}}=0
$$


whose two solutions are the functions $\chi=e^{\frac{1}{2} f}$ and $\chi=e^{\frac{1}{2} t}$. Consequently, the quantization space $\mathcal{H}_{0}$ is

$$
\mathcal{H}_{0}=\left\{1 \otimes(\chi \beta) \in \Gamma(K \otimes \mathcal{D}) /-\frac{\partial \chi}{\partial \bar{z}_{k}}+\frac{\partial f}{\partial \bar{z}_{k}} \frac{\partial \chi}{\partial t}+\frac{\chi}{2} \frac{\partial f}{\partial \bar{z}_{k}}=0, \quad \forall k=1, \ldots, n\right\} \neq\{0\} .
$$

For the elements of $\mathcal{H}_{0}$ and for $g \in \mathcal{Q}(\mathcal{P})$, taking into account (22) and (35), we obtain the quantum operator

$$
\hat{g}(1 \otimes(\chi \beta))=\left(2 \pi i g \chi+\Lambda(d g, d \chi)+\frac{\chi}{2} \operatorname{div} \Lambda^{\sharp}(d g)\right)(1 \otimes \beta) .
$$

Furthermore, the inner product of two elements $1 \otimes\left(\chi_{1} \beta\right), 1 \otimes\left(\chi_{2} \beta\right)$ of $\mathcal{H}_{0}$ with compact support is

$$
\left\langle 1 \otimes\left(\chi_{1} \beta\right), 1 \otimes\left(\chi_{2} \beta\right)\right\rangle=\int_{M} \chi_{1} \bar{\chi}_{2} v .
$$

\section{References}

[1] Aschieri, P., Baković, I., Jurčo, B., Schupp, P., "Noncommutative gerbes and deformation quantization" (arXiv:hep-th/0206101).

[2] Bates, S., Weinstein, A., Lectures on the Geometry of Quantization (A.M.S., Berkeley Mathematics Lecture Notes Series, Providence, 1997).

[3] Cannas da Silva, A., Weinstein, A., Geometric Models for Noncommutative Algebras (A.M.S., Berkeley Mathematics Lecture Notes Series, Providence, 1999).

[4] Catanneo, A.S., Xu, P., "Integration of twisted Poisson structures," J. Geom. Phys. 49, 187-196 (2004).

[5] Cornalba, L., Schiappa, R., "Nonassociative Star Product Deformations for Dbrane Worldvolumes in Curved Backgrounds," Commun. Math. Phys. 225, 33-66 (2002).

[6] Huebschmann, J., "Poisson cohomology and quantization," J. Reine Angew. Math. 408, 57-113 (1990).

[7] Klimčík, C., Ströbl, T., "WZW-Poisson manifolds," J. Geom. Phys. 43, 341-344 (2002).

[8] Kobayashi, S., Nomizu, K., Foundations of Differential Geometry (Wiley, New York, 1969).

[9] Kosmann-Schwarzbach, Y., Laurent-Gengoux, C., "The modular class of a twisted Poisson structure," Travaux Mathématiques 16, 315-339 (2005).

[10] Kosmann-Schwarzbach, Y., Milen, Y., "Modular classes of regular twisted Poisson structures on Lie Algebroids," Lett. Math. Phys. (to be published), math.SG/0701209.

[11] Kostant, B., "Quantization and Unitary Representations," in Lectures in Modern Analysis and Applications III, edited by Taam, C.T., Lecture Notes in Math. 170 (Springer, Berlin, 1970), pp. 87-207. 
[12] Koszul, J.L., "Crochet de Schouten-Nijenhuis et cohomologie," in Élie Cartan et les Mathématiques d'aujourd'hui, Astérisque, Numéro Hors Série (1985), pp. 257271.

[13] de León, M., Marrero, J.C., Padrón, E., "On the geometric quantization of Jacobi manifolds," J. Math. Phys. 38 (12), 6185-6213 (1997).

[14] Marle, Ch.-M., "De la mécanique classique à la mécanique quantique: pourquoi et comment quantifier?" in Feuilletages-Quantification géometrique, (Maison des Sciences de l'Homme, Paris, 2003), pp. 1-18. (http://perso.orange.fr/CharlesMichel.Marle/)

[15] Nunes da Costa, J.M., Petalidou, F., "Twisted Jacobi manifolds, twisted DiracJacobi structures and quasi-Jacobi bialgebroids," J. Phys. A: Math. Gen. 39, 10449-10475 (2006).

[16] Park, J.S., "Topological open p-branes," in Symplectic geometry and Mirror Symmetry, eds. Fukaya, K., Oh, Y.G., Ono, K., Tian, G., (Seoul, 2000), (World Sci. Publishing, River Edge, NJ, 2001), pp. 311-384.

[17] Petalidou, F., "Prequantizable twisted Poisson manifolds and twisted Jacobi structures," (in preparation).

[18] Ševera, P., Weinstein, A., "Poisson geometry with a 3-form background," in Proceedings of the International Workshop on Noncommutative Geometry and String Theory, Prog. Theor. Phys. Suppl. 144, 145-154 (2001).

[19] Ševera, P., "Quantization of Poisson Families and of Twisted Poisson Structures," Lett. Math. Phys. 63, 105-113 (2003).

[20] Śniatycki, J., Geometric Quantization and Quantum Mechanics (Springer, Berlin, 1980).

[21] Souriau, J.M., Structures des Systèmes Dynamiques (Dunod, Paris, 1969).

[22] Sternberg, S., Lectures on Differential Geometry (Prentice-Hall, Englewood Cliffs, 1964).

[23] Vaisman, I., "Basic ideas of geometric quantization," Rend. Sem. Mat. Torino 37, 31-41 (1979).

[24] Vaisman, I., "On the geometric quantization of Poisson manifolds," J. of Math, Physics 32 (12), 3339-3345 (1991).

[25] Vaisman, I., Lectures on the Geometry of Poisson Manifolds, Progress in Math. 118 (Birkhauser, Basel, 1994).

[26] Yano, K., The theory of Lie derivatives and its applications (North Holland Publ., Amsterdam, 1957).

[27] Weinstein, A., Xu, P., "Extensions of symplectic groupoids and quantization," J. Reine Angew. Math. 417, 159-189 (1991).

[28] Weinstein, A., Zambon, M., "Variations on Prequantization," Travaux Mathématiques 16, 187-219 (2005).

[29] Woodhouse, N., Geometric Quantization (Claredon, Oxford, 1980). 